Dengue Fever:

\title{
An Emerging Infectious Disease in The Bahamas
}

\author{
Sherrie V. Bain \\ Keiser University ${ }^{1}$
}

\section{ABSTRACT}

Dengue fever, an emerging infectious disease, is increasing in prevalence in many geographic regions, including the Caribbean. It is the most common arboviral (vector-borne) disease in the world, and infects more that 50 million people annually worldwide. The etiological agent of dengue fever is one of four serotypes of the dengue virus (DENV1-DENV4). The infection is transmitted via a human-mosquito-human route, when one or more species of the Aedes mosquito takes a blood meal from an infected host and then feeds on a person who is uninfected. There is no vaccine or cure for dengue fever. Dengue fever is a growing cause for concern in The Bahamas. This year the incidence of dengue fever reached epidemic proportions in The Bahamas. This article will explore the etiology and epidemiology of dengue fever, and offer some insight into how The Bahamas can in the future begin to develop strategies for the eradication of dengue fever.

\section{INTRODUCTION}

Emerging infectious diseases are new diseases that have not been known to cause infections in humans before, as well as diseases that have been present in the human population for a long period of time, but were previously limited to a few endemic regions. However, due to factors such as the ones that will be discussed in this article, emerging infectious diseases are dramatically increasing in incidence globally (Tortora, Funke, \& Case, 2010). These types of diseases are of great concern from a public health perspective because there are often few cures available to prevent or treat them. In addition, the spread of such diseases to new geographic regions where they are not endemic often places a strain on the public health sector whenever there is a new disease outbreak. Healthcare facilities may have limited resources available to treat outbreaks of emerging infectious diseases. They often become overwhelmed in an effort to treat large numbers of new cases in patients who have very little natural immunity to these infections. Last year in Haiti Vibrio cholera caused over 100,000 cases of cholera, including more than 3,000 fatalities. Prior to the 2010 epidemic, cholera had not been observed in this small island nation in over a century (Gorry, 2011).

This year, The Bahamas is experiencing a dengue fever outbreak of epic proportions.

\footnotetext{
${ }^{1}$ Sherrie V. Bain, Adjunct Microbiology Instructor, Keiser University, 400 Rome Drive, E318, Palm Springs, FL 33461.

E-mail: svbain@yahoo.com

How to cite this article in APA (6 ${ }^{\text {th }}$ ed. Style): Bain, S. V. (2011). Dengue fever: An emerging infectious disease in The Bahamas. The International Journal of Bahamian Studies, 17(2), 67-72. https:// doi.org/10.15362/ijbs.v17i2.155
}

CS. V. Bain, 2011. Journal compilation (CThe International Journal of Bahamian Studies, 2011. 
Although there have not been any confirmed cases of fatalities directly linked to the dengue fever outbreak, the public health sector is struggling to keep up with the number of new cases of dengue fever. This is a disease outbreak that is caused by a pathogen that is not historically endemic to The Bahamas. There is currently widespread concern among the general public about dengue fever, how it is contracted, and why it is suddenly ravaging The Bahamas. There is also grave concern about the economic impact that the current dengue fever outbreak might have on The Bahamas due to loss of productivity and loss of tourism revenue (Recognizing the seriousness of dengue fever, 2011). This is not the first time that a dengue fever outbreak has occurred in The Bahamas. Dengue fever outbreaks also occurred in 1998 and 2003 (Figure 1). However, the number of confirmed cases in this year's outbreak (as of October 2011) is almost seven times that of the last two dengue fever outbreaks combined (Turnquest, 2011).

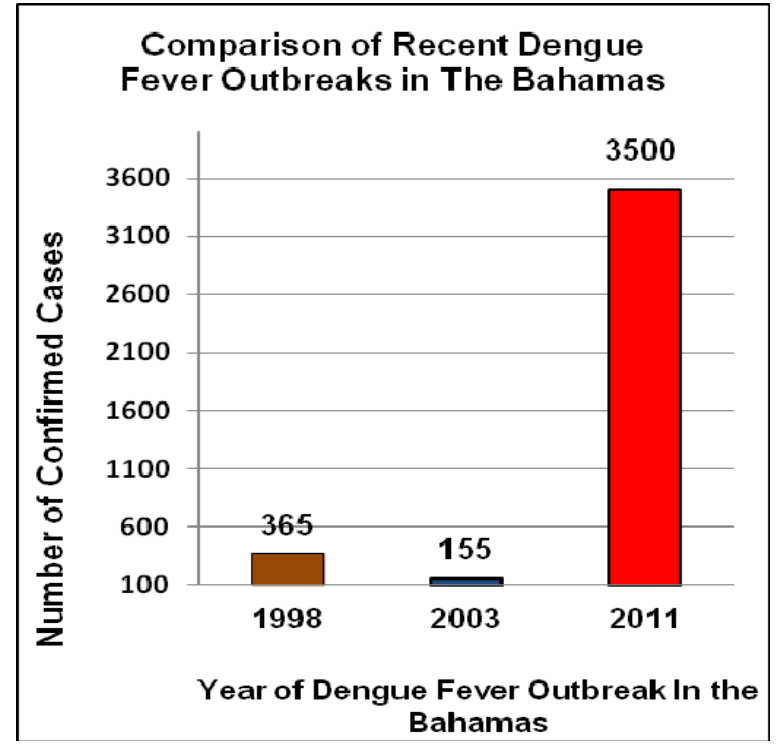

Figure 1. Comparison of dengue fever outbreaks in The Bahamas between 1998 and September 2011. The data shown represent the number of confirmed cases of dengue fever during the 1998 (365 cases), 2003 (155 cases) \& 2011 (>3500 cases) outbreaks. The estimated number of confirmed dengue fever cases in the current outbreak is predicted to be significantly higher.
The actual number of dengue fever cases in the current outbreak is estimated to be significantly higher. However, at the height of the dengue fever outbreak, public health facilities were being overwhelmed with persons seeking treatment for dengue fever symptoms. As a result some patients were being treated and sent home without an actual serological test to confirm the dengue virus. In addition, this 2011 outbreak includes several cases of dengue hemorrhagic fever (DHF), an acute form of dengue fever, which may result in severe hemorrhaging and possible fatalities (Rolle, 2011).

This disease trend suggests that dengue fever is becoming more prevalent in The Bahamas. Therefore, unless there are significant developments at the national and/or international levels, aimed at the eradication of dengue fever, the impact might be even more severe during future outbreaks. This is consistent with the dengue fever trends in other geographic locations as well (World Health Organization, 2009). At the national level, The Bahamas will need a multi-faceted, pre-emptive approach that is not simply limited to vector control at the onset of the next outbreak.

\section{ETIOLOGY AND EPIDEMIOLOGY OF DENGUE FEVER}

In order to begin to develop a national strategy for the eradication of dengue fever in The Bahamas, one must first address the etiology and epidemiology of dengue fever. Dengue fever is caused by the dengue virus (DENV). The dengue virus is a member of the Flaviviridae family. These viruses possess a single, plus-strand RNA genome, enclosed in an icosahedral capsule. Other members of this family include the hepatitis $\mathrm{C}$ virus and yellow fever virus. Viruses like hepatitis $\mathrm{C}$ are referred to as oncogenic viruses because they can sometimes transform normal cells into tumor cells upon infection. Whereas viruses such as the yellow fever 
virus and dengue virus are categorized as arboviruses or hemorrhagic fever viruses because they are transmitted by arthropod vectors, and cause hemorrhagic fevers that primarily affect the cardiovascular system. There are four serotypes of dengue viruses that cause infections in humans. These are referred to as DENV-1, DENV-2, DENV-3 and DENV-4 (Pommerville, 2011).

The arthropod vector for the dengue virus is the Aedes spp. mosquito. Two species of the Aedes mosquito are known to transmit the dengue virus to humans. These are Aedes aegypti and Aedes albopictus. In addition to the human form of dengue fever there is also a zoönotic form of the disease that exists in monkeys and requires a different species of the Aedes mosquito for transmission. There is no known case of overlap or inter-species transmission of the human dengue and simian dengue fever diseases (Pourrut, Nkoghé, Gonzalez, \& Leroy, 2011). Humans become infected with the dengue virus when a female Aedes spp. mosquito takes a blood meal from an infected human host, and then bites another uninfected human, thereby transmitting the virus during the second feeding event (Figure 2). Thus, humans are in fact a natural reservoir for the dengue virus and play a critical role in the spread of dengue virus to new geographic regions.

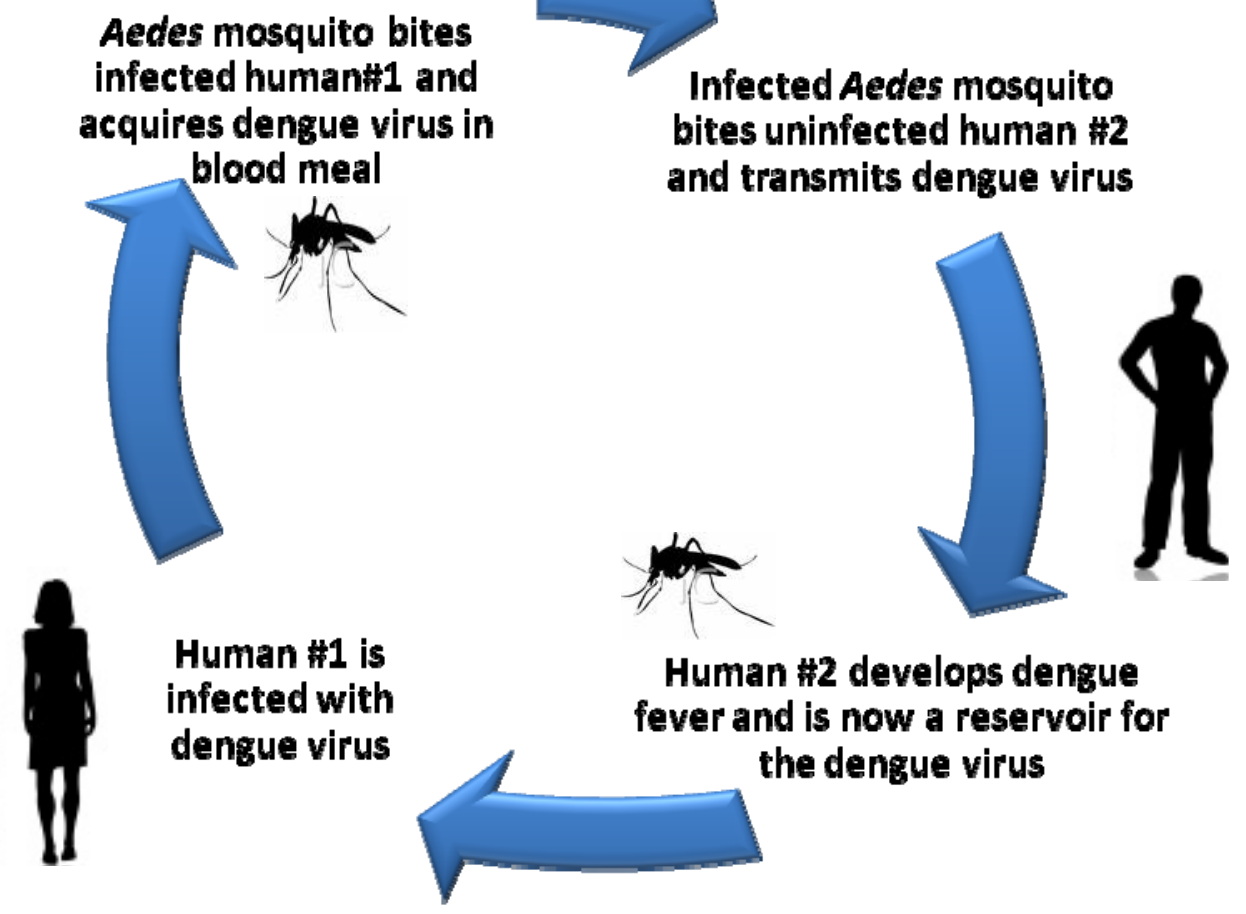

Figure 2. Disease transmission cycle for the human form of dengue fever. Both the mosquito vector and human reservoir are crucial to maintaining this disease cycle. Humans are the only known mammalian reservoir of the human-associated dengue fever virus. This transmission cycle is completely separate from the zoönotic dengue fever transmission cycle affecting monkeys. 
Infection with one type of the dengue virus will typically provide lifelong immunity against that serotype. However, it does not provide lasting immunity against the other three serotypes of the dengue virus. Therefore, even if a person has had dengue fever before, they can still become re-infected with another serotype of the dengue virus. Most cases of dengue fever are limited to severe flu-like symptoms that can be managed with supportive care. The infected person usually recovers in approximately two weeks after the onset of the disease symptoms. However, in a growing number of incidences, persons who become infected with the dengue virus experience a more severe complication called dengue hemorrhagic fever (DHF). This form of the illness can be fatal, especially for individuals who are immune-compromised. There is no clear scientific evidence to suggest a correlation between recurring dengue fever infections and the progression to DHF. Recently, the World Health Organization reported a more than four-fold increase in the number of countries where the incidence of DHF has significantly increased over a 25-year period (World Health Organization, 2009).

Historically, dengue fever and the Aedes mosquitoes that transmit it were limited to tropical and sub-tropical regions of the world. However, that is rapidly changing due to human migration and global human interaction. Humans are the natural reservoir for the dengue virus and whenever infected humans travel or migrate from one region of the world to another, there is a possibility of spreading the dengue virus to new areas of the world, if the mosquito vector is also present in that new location (Dengue epidemiology, 2011). Although The Bahamas is a country in a sub-tropical region of the world, it has not historically been a country where dengue fever is endemic. However, if the disease pattern continues to establish itself, The
Bahamas will become one of the growing numbers of countries where dengue fever is now endemic (World Health Organization, 2009).

In the case of the elevated incidence of dengue fever in The Bahamas, Bahamians who have traveled to regions where dengue fever is endemic, might have become infected and then returned home to The Bahamas to inadvertently become the human reservoirs that are driving the establishment of dengue in the country. In addition, visitors and immigrants to The Bahamas from regions where dengue fever is endemic have also played a role in this disease cycle. As with the expansion of human populations, environmental changes in global climate patterns are also increasing the ecological habitat range of the Aedes mosquitoes that transmit the dengue virus. The Aedes mosquito is very common in The Bahamas, thus the presence of the vector and the growing presence of human reservoirs may mean that the 2011 dengue fever outbreak in The Bahamas is just the tip of the iceberg.

\section{MOVING TOWARD A DENGUE FEVER ERADICATION STRATEGY}

The Bahamas is now facing a turning point in relation to the relevance of dengue fever as a public health crisis and potential detriment to the economic viability of the country. It is time for the public health sector, as well as The College of The Bahamas to begin to take measures to develop a comprehensive dengue fever eradication policy in The Bahamas. Although reduction of the Aedes vector population by using insecticides may seem like the obvious route to pursue, it may also prove to be the most difficult goal to accomplish. The effects of such vector control measures are often transient. As with other tropical and sub-tropical regions of the world where vector-borne diseases such as malaria and yellow fever continue to ravage entire countries, eliminating a disease vector 
such as the Aedes or Anophles mosquito is proving to be extremely difficult. Even when chemical insecticides are used, the impact on the mosquito population is not permanent. The Aedes mosquito breeds in urban areas such as trash sites and old tires with standing water, as well as in natural habitats such as mangrove swamps. Especially in a country like The Bahamas with its vast natural habitat for mosquitoes, application of insecticides may prove ineffective in dengue virus control in the long term. In addition, there are concerns about the environmental impact of sustained use of insecticides to provide ongoing control of the mosquito population (World Health Organization, 2009).

It is important to note that the problem of vector control/eradication is a global problem that is not just limited to the current crisis in The Bahamas. In addition to the lack of an effective vector eradication strategy, researchers are also discovering that there is both a positive and negative correlation between vector populations and the incidence of dengue fever in some parts of the world. In other words, having a low density of the mosquito population does not always correlate to having a lower incidence of the disease within a specific geographic region (Lin \& Wen, 2011). Therefore, The Bahamas should look beyond simply trying to control the vector population as a lasting solution to eradicating dengue fever. These alternative strategies need to include researching how the disease is transmitted in the mosquito vector, as well as how it may be prevented from becoming established in its human hosts (Xi, Ramirez, \& Dimopoulos, 2008).

The College of The Bahamas in particular, can be a key innovator in developing these alternative strategies. As the College of The Bahamas moves towards university status, emphasis needs to be placed on developing a national infectious disease research center that will focus primarily on discovering drug and vaccine targets against diseases such as dengue fever and HIV. Such diseases threaten the public health and socio-economic well-being of the entire country. However, The College of The Bahamas cannot shoulder the monumental task of bringing The Bahamas to the pinnacle of infectious disease research and innovation alone. This is an objective that will require the participation and partnership of multiple governmental and private organizations within The Bahamas. The Public Health Authority, including the Princess Margaret Hospital and the Department of Environmental Health, will have to share intellectual and material resources to successfully establish a national infectious disease research center in The Bahamas.

In addition, there are many private organizations such as the Lyford Cay Foundation, the Kiwanis Club, the Rotary Club, the AIDS Foundation of The Bahamas, and the Bahamas Red Cross who, through their established philanthropic and public health-related activities, can act as advocates and patrons for the development of such an important national asset. Despite the historical lack of emphasis on the pursuit of scientific research to identify potential vaccine targets against infectious diseases such as dengue fever, there is a growing urgency for The Bahamas to now give greater attention to these endeavours. The Bahamas could become a regional leader in finding effective cures and treatments for diseases such as dengue fever. Not only would a national infectious disease research center be of great benefit to the country from a public health perspective, but it would also provide the infrastructure to train and retain Bahamian scientists who wish to pursue careers in infectious disease research, but who currently have to do so at institutions and organizations overseas. 


\section{REFERENCES}

Dengue epidemiology. (2011). http://www.denguevirusnet.com/epidemiol ogy.html

Gorry, C. (2011). Haiti one year later: Cuban medical team draws on experience and partnerships. MEDICC Review , 13(1), 5255.

http://www.medicc.org/mediccreview/artic les/haiti-one-year-later.pdf

Lin, C.-H., \& Wen, T.-H. (2011). Using geographically weighted regression (GWR) to xxplore spatial varying relationships of immature mosquitoes and human densities with the incidence of dengue. International Journal of Environmental Research and Public Health, 8(7), 279-281. http:// www.mdpi.com/16604601/8/7/2798/

Pommerville, J. C. (2011). Alcamo's fundamentals of microbiology. (9th ed.). Jones and Bartlett.

Pourrut, X., Nkoghé, D., Gonzalez, J.-P., \& Leroy, E. (2011). No evidence of dengue virus circulation in Rural Gabon. [Letter to the Editor]. Emerging Infectious Diseases, 17(8), 1568-1569.

https://doi.org/10.3201/eid1708.110153
Rolle, K. (2011, July 29). 195 cases of dengue fever confirmed. The Nassau Guardian. http://www.thenassauguardian.com/index. php?option=com_content\&view=article\&i $\mathrm{d}=11871 \&$ Itemid $=27$

Recognizing the seriousness of dengue fever [Editorial]. (2011, August 19). The Freeport News. http://freeport.nassauguardian.net/editorial/ 378346027766432.php

Tortora, G. J., Funke, B. R., \& Case, C. L. (2010). Microbiology: An introduction (10th ed.). Pearson Benjamin Cummings.

Turnquest, A. (2011, September 19). US media: Two children contract dengue fever on Bahamas vacation. The Tribune, A1. http://www.tribune242.com/searchresults/0 9192011_denguefever_news_pg1

World Health Organization. (2009). Dengue and dengue haemorrhagic fever. http:// www.who.int/mediacentre/factsheets / fs117/en/

Xi, Z., Ramirez, J. L., \& Dimopoulos, G. (2008). The Aedes aegypti toll pathway controls dengue virus infection. PLoS Pathogens , 4(7), 1-12. https://doi.org/10.1371/ journal.ppat.1000098 